\title{
Infección diseminada por adenovirus de curso fatal en un niño inmunocomprometido
}

\author{
Katia Abarca V., Nicole Le Corre P., Cecilia Perret P., Angélica Wietstruck P., \\ David Oddó B. y Francisco Barriga C.
}

\section{Disseminated and fatal adenovirus infection in an immunocompromised child}

Severe adenovirus (ADV) infections have become increasingly important in immunocompromised patients, mainly in pediatric stem cell transplant recipients. We report a case of disseminated ADV infection leading to death in a 12-year-old stem cell transplant recipient. The diagnosis was confirmed by viral isolation and viral genome detection in tissues and blood. Main issues associated with infection, diagnosis and therapeutic alternatives are reviewed. This case should alert clinicians to suspect and study this agent in high risk patients and highlights the importance of having antiviral drugs for ADV available in Chile.

Key words: Adenovirus infection, human, immunocompromised host, bone marrow transplantation.

Palabras clave: Infección adenovirus, humana, paciente inmunocomprometido, trasplante médula ósea.

\author{
Pontificia Universidad Católica \\ de Chile \\ Escuela de Medicina \\ Departamento de Pediatría \\ (KAV, NLCP, CPP, AWP, FBC) \\ Departamento de Anatomía \\ Patológica (DOB)
}

Recibido: 6 de julio 2007 Aceptado: 15 de noviembre de 2007

Correspondencia a: Katia Abarca Villaseca katia@med.puc.cl

\section{Introducción}

A denovirus es un virus endémico en la población general, que afecta predominantemente a niños entre seis meses y cinco años de edad, causando infecciones respiratorias, oculares o gastrointestinales, generalmente autolimitadas. Ocasionalmente puede presentarse como infecciones graves en pacientes inmunocompetentes ${ }^{1-3}$. Con creciente frecuencia se han reconocido, en los últimos años, infecciones graves en individuos inmunocomprometidos, en especial en pacientes sometidos a trasplante de precursores hematopoyéticos $(\mathrm{TPH})^{4-7}$.

Se reporta el caso de un niño con infección diseminada por ADV de curso fatal y una revisión de la literatura de los principales aspectos de la infección, su diagnóstico y posibilidades terapéuticas.

\section{Caso Clínico}

Escolar de sexo masculino, con 12 años de edad, sometido a TPH de sangre de cordón de donante no relacionado, por leucemia linfoblástica aguda en tercera remisión. Recibió radioterapia corporal total en el acondicionamiento y con posterioridad al trasplante, prednisona y en forma secuencial tacrolimus y ciclosporina.

Alrededor del día +60 post TPH inició un cuadro caracterizado por náuseas y decaimiento, sin fiebre, dolor abdominal, vómitos ni alteración de las deposi- ciones. Por la persistencia de las náuseas se hospitalizó para estudio, resultando negativos los hemocultivos, antigenemia para CMV, coprocultivo, copro-parasitológico, rotavirus y toxina de Clostridium difficile. Se le realizó una endoscopia digestiva alta que mostró cambios inflamatorios macroscópicos en el duodeno, con histología normal. Persistió su estado nauseoso y se agregó distensión abdominal y constipación alternante. Al día +79 presentó fiebre hasta $39{ }^{\circ} \mathrm{C}$, diarrea sin sangre y dolor abdominal tipo cólico por lo que se repitieron los estudios microbiológicos y se le realizó una TAC de abdomen, resultando todos normales, a excepción de la antigenemia para CMV positiva (2 núcleos en 400.000 células) por lo que se inició terapia con ganciclovir. Persistió con gran decaimiento y náuseas, catalogadas por el niño como "muy intensas", que le impedían alimentarse, indicándosele nutrición parenteral total el día +87 . De sus exámenes destacaban linfopenia (442 linfocitos $/ \mathrm{mm}^{3}$ ) y elevación moderada de enzimas hepáticas (SGOT 208 mg/dL, SGPT 282 $\mathrm{mg} / \mathrm{dL}, \mathrm{GGT} 181 \mathrm{mg} / \mathrm{dL})$, con bilirrubinemia y protrombinemia normales. La antigenemia para CMV se negativizó a los 3 días. Se le realizó una nueva endoscopia digestiva alta que demostró una duodenitis erosiva. Por sospecha de estar frente a una enfermedad injerto contra huésped (EICH) se inició tratamiento con mesalazina. La biopsia intestinal mostró numerosos enterocitos con inclusiones intranucleares tipo Cowdry A y efecto citopático, compatibles con infección viral (Figura 1). A raíz de estos hallazgos se solicitó cultivo de deposiciones para CMV, ADV y VHS, 
Figura 1. Biopsia de intestino delgado. Numerosos enterocitos con típicas inclusiones intranucleares tipo Cowdry A (flechas) y algunas de tipo célula borrosa (punta de flecha), efecto citopático compatible con la infección por ADV (hematoxilina \& eosina; $X 500$ aumento original).

Figura 2. TAC de abdomen que muestra múltiples lesiones focales en el parénquima hepático, hipodensas, multiloculadas, de contornos lobulados, probablemente de causa inflamatoria-infecciosa (abscesos). La de mayor tamaño se ubica en el segmento posterior del lóbulo derecho (flecha).

Figura 3. Biopsia hepática. 3A: célula hepática con una típica inclusión intranuclear basófila con halo claro, tipo Cowdry A (flecha), efecto citopático compatible con la infección por ADV (hematoxilina \& eosina; X 1250 aumento original). $3 \mathrm{~B}$ : dos células hepáticas con típicas inclusiones intranucleares basófilas grandes que llenan los núcleos y se extienden al citoplasma, tipo célula borrosa (flecha); efecto citopático característico de la infección por ADV (hematoxilina \& eosina; $X$ 1250 aumento original).
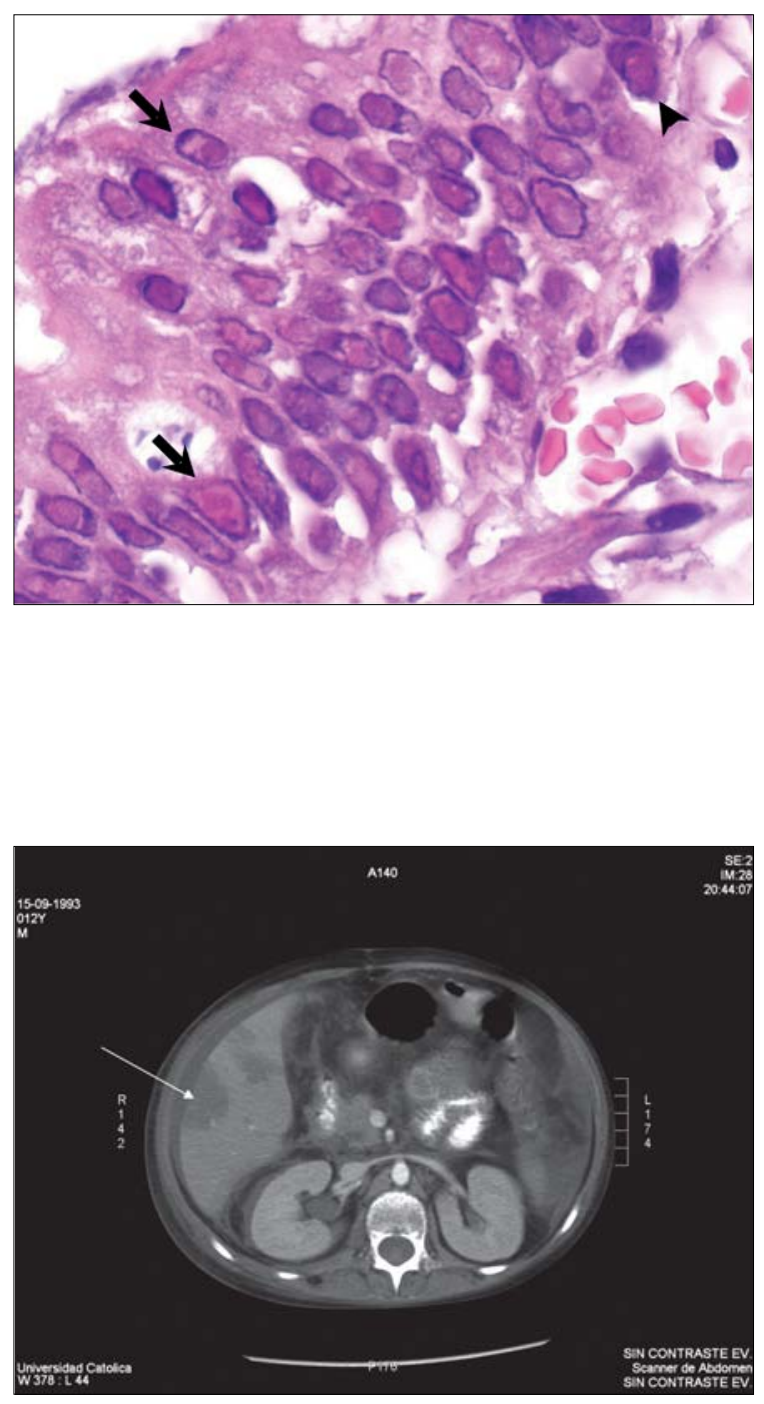

resultando positivo el cultivo de ADV por técnica de shell vial. Con la sospecha de una enfermedad diseminada se intentó detectar el genoma de ADV por RPC en sangre, con resultado positivo. Una TAC abdominal mostró lesiones hipodensas hepáticas sugerentes de abscesos (Figura 2). Un estudio de hisopado nasofaríngeo por IFD demostró co-infección por virus parainfluenza 3. La biopsia hepática, efectuada por punción bajo ecoscopia, fue informada como células hepáticas con inclusiones intranucleares basófilas grandes, efecto citopático compatible con de infección por ADV (Figura 3). El cultivo hepático fue positivo para ADV y negativo para bacterias (incluyendo micobacterias), hongos y otros virus. Se mantuvo sin neutropenia, pero con linfopenia progresiva (hasta $185 / \mathrm{mm}^{3}$ ) con ausencia de LT y recuento absoluto de LB disminuido. Dada la confirmación de infección diseminada por $\mathrm{ADV}$, se inició terapia con rivabirina vía oral en espera de procurar cidofovir. En los días siguientes presentó agravamiento progresivo, con aumento sostenido de las enzimas hepáticas (SGOT 596-7.792 mg/ dL, SGPT 468-485 mg/dL, GGT 181-1.185 mg/dL, LDH 649-10.200 mg/dL), y aparición de infiltrado pulmonar intersticial confluente bilateral, con detección de ADV por IFD en un hisopado nasofaríngeo. Al momento de conseguirse el cidofovir, el niño se encontraba con importante deterioro respiratorio y hepático, los cuales progresaron a pesar de todas las medidas tomadas, falleciendo al tercer día de tratamiento y a cuarenta días de iniciados los síntomas digestivos. Posteriormente se realizó tipificación del virus por RPC en el Laboratorio de Virología de la Universidad de Chile, amplificando una zona del gen de la fibra con partidores para los grupos B y C, con resultado negativo para ambos $^{8}$.
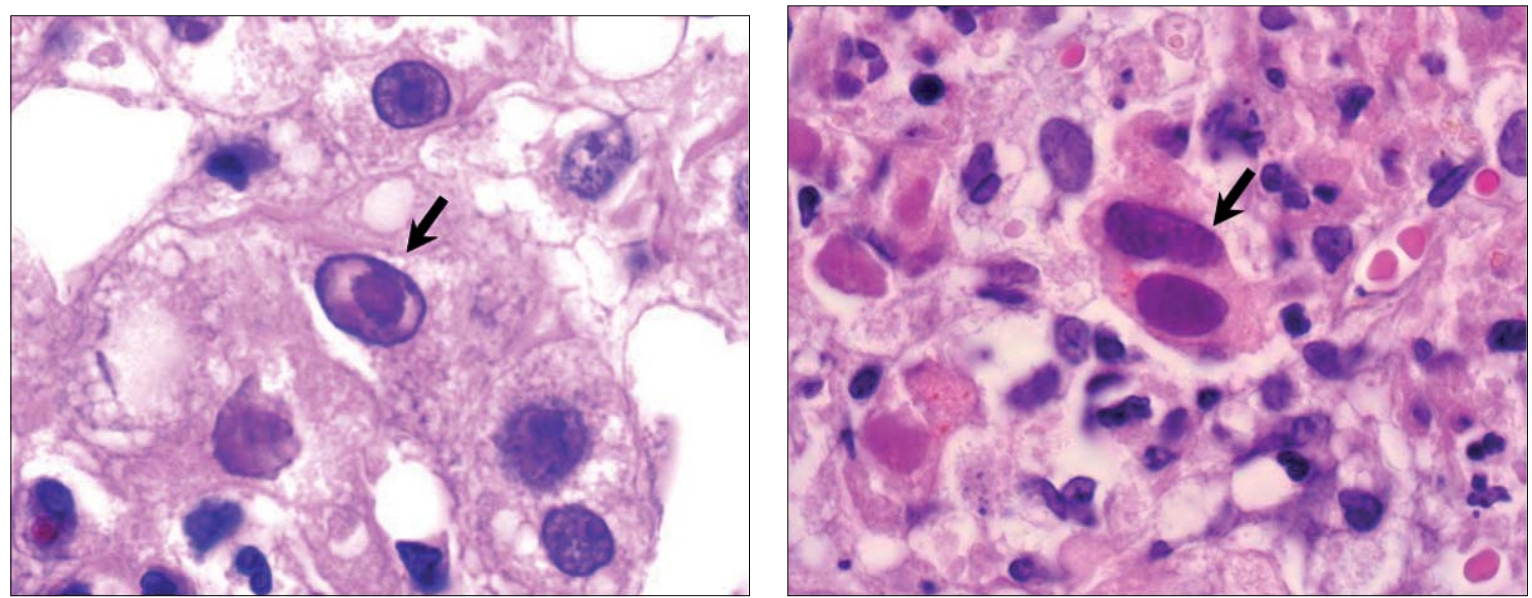


\section{Discusión}

Las infecciones por ADV en la población que es previamente sana tienen, generalmente, un curso autolimitado. Ocasionalmente, ADV es causa de infecciones respiratorias graves en niños inmunocompetentes y brotes en centros hospitalarios; en estas situaciones se asocia a graves secuelas pulmonares ${ }^{1,3,9,10}$.

En pacientes inmunocomprometidos, especialmente aquellos sometidos a TPH, es causa importante de infecciones que pueden llegar a ser graves por su diseminación y compromiso multisistémico. En ellos, es el tercer virus más frecuente después de CMV y VHS ${ }^{4}$; con la mejoría de los métodos diagnósticos, terapia y profilaxis de estos agentes, ADV ha pasado a ser un patógeno de alta importancia y creciente reco-

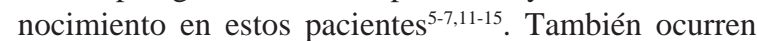
infecciones graves por ADV en pacientes con otros tipos de inmunodeficiencias como las congénitas, receptores de órganos sólidos, pacientes tratados con corticosteroides o SIDA ${ }^{16-19}$.

Existen 51 serotipos de ADV reconocidos en infecciones en humanos, clasificados en seis grupos (A-F). Los serotipos de los grupos A, B y C son los más frecuentemente asociados a infecciones en hospederos inmunocomprometidos ${ }^{15,18-20}$. En Chile, en niños hospitalizados por infecciones respiratorias bajas los grupos más frecuentes son $\mathrm{B}$ y C; el serotipo 7h se ha encontrado asociado a los cuadros más graves ${ }^{3,9}$. En este caso clínico sólo se pudo determinar que no correspondía a los grupos B ni C.

La incidencia descrita de infección por ADV post TPH varía entre 3 y 47\% ${ }^{4-7,12-14,21}$. Aproximadamente $10 \%$ de ellas en adultos y hasta $25 \%$ en niños pueden ser asintomáticas ${ }^{22}$. La ausencia de síntomas o una clínica inespecífica hacen que con alta frecuencia las infecciones no sean reconocidas sino hasta etapas avanzadas $^{15,22}$.

Se han descrito varios factores de riesgo asociados a infección por ADV en TPH: edad (en niños la frecuencia es, al menos, tres veces mayor que en adultos) ${ }^{11}$, presencia de EICH moderada a grave ${ }^{4-6}$, tipo de trasplante (más frecuente en alogeneico, principalmente en trasplante de donante no relacionado $)^{15}$, depleción de células $T^{7,21}$, recuentos de linfocitos CD4 bajos (menor a 300 céls $/ \mathrm{mm}^{3}$ ) a los 100 días post $\mathrm{TPH}^{21,23-25} \mathrm{y}$ dador positivo para $\mathrm{ADV}^{13}$. Con la excepción de EICH moderada a grave y del estado del donante para ADV (cordón de recién nacido y por tanto negativo para ADV), nuestro paciente presentaba todos los factores de riesgo mencionados.

La frecuencia de infección diseminada por ADV en pacientes sometidos a TPH varía entre 0,9 y 9,6\% en niños $^{5,6}$. En estos casos, la tasa de mortalidad llega a
$50-60 \%{ }^{15,16,24}$, siendo más alta en los pacientes que desarrollan neumonía o hepatitis ${ }^{5,18,26}$. Otros sitios que se afectan con frecuencia son la vejiga (cistitis hemorrágica), tracto gastrointestinal (colitis) y SNC ${ }^{15}$. En una serie ${ }^{11}$ ADV fue detectado inicialmente en las deposiciones en $83 \%$ de los pacientes. Muchas veces el aparato gastrointestinal corresponde al sitio inicial de la afección para una posterior diseminación ${ }^{6}$, tal como ocurrió en nuestro paciente.

La hepatitis por ADV en TPH es inusual, ocurre en 0,2 a $0,5 \%$ de los casos, siendo la hepatitis fulminante de muy baja ocurrencia ${ }^{5,6,11,15,18,27}$. Se han reportado algunos casos de hepatitis fulminante por ADV en pacientes inmunosuprimidos por quimioterapia ${ }^{28,29}$ y en pacientes con SIDA ${ }^{17}$. El compromiso hepático grave es más frecuente en pacientes sometidos a trasplantes hepáticos, en quienes ocurre con una frecuencia de 8 a 10\%; 2,2\% cursan con hepatitis fulminante ${ }^{30}$. El serotipo 5 es el más frecuente en pacientes con compromiso hepático ${ }^{16,19}$. En muy pocos casos se han reportado lesiones necróticas hepáticas ${ }^{17,29} \mathrm{y}$, en nuestro conocimiento, no se han descrito casos con imágenes sugerentes de abscesos hepáticos causados por ADV. La identificación de ADV por cultivo en biopsia hepática tomada de una de esas lesiones y el estudio negativo para otros patógenos confirma a este agente como la causa de las lesiones hepáticas con aspecto de abscesos que presentó nuestro paciente. Varios autores coinciden en el mal pronóstico de los pacientes con compromiso hepático ${ }^{5,18,26}$.

La aparición de síntomas de la infección desde el día en que se efectúa el TPH es variable, con una mediana de 54 días y un rango de 4 a 333 días ${ }^{15,26}$. En niños ocurre más frecuentemente en los primeros 30 días, mientras que en adultos suele manifestarse después de los 90 días $^{6}$. Algunos autores sugieren que los niños padecen más de infecciones primarias por ADV, mientras que los adultos presentarían principalmente reactivación de infecciones latentes ${ }^{15}$. Se ha demostrado latencia de ADV en células mononucleares periféricas, pulmones y tracto respiratorio superior ${ }^{15,31}$.

La respuesta inmune no es bien conocida, pero parece ser importante la respuesta de células $\mathrm{T}$ para la recuperación. La sobrevida de los pacientes se asocia con aumento de linfocitos en las primeras semanas post TPH y el control de la infección viral se correlaciona con la presencia de LT CD4 específicos ${ }^{21,32}$. Según los autores, la reconstitución linfocitaria es crucial para la recuperación clínica, la disminución de la viremia y la sobrevida de los pacientes. Concordante con esto, en la evolución de nuestro paciente destaca la linfopenia prolongada y la ausencia de linfocitos T. Parece lógico concluir que este niño no logró una reconstitución linfocitaria que le permitiera controlar la infección. 
Los métodos diagnósticos para confirmar una infección por ADV son el cultivo tradicional que demora entre 3 y 21 días, el cultivo acelerado (shell vial), la identificación directa en secreciones respiratorias u orina por ELISA o IF, la observación de partículas virales características por microscopia electrónica (ME) en orina o deposiciones y la serología en individuos inmunocompetentes ${ }^{15}$.

Las técnicas moleculares, de gran desarrollo en los últimos años, han permitido un mejor estudio de los pacientes. Actualmente, en muchos centros se encuentra disponible la detección de genoma viral por RPC en sangre, deposiciones, orina y LCR ${ }^{13,15}$.

Los criterios considerados diagnósticos de infección por ADV son: detección de virus por ME, cultivo, histología o RPC de tejido estéril (excluyendo tracto GI) de un paciente con síntomas compatibles y sin otra causa identificada. Se considera infección definitiva la demostración del virus por histopatología o cultivo de tejidos y probable, aquella demostrada por cultivo de dos o más muestras diferentes a biopsias ${ }^{6,33}$. Una infección por ADV es considerada diseminada cuando hay compromiso multiorgánico incluyendo hígado y pulmón ${ }^{34}$.

Varios autores han observado que la detección de ADN viral en sangre parece ser un marcador de progresión a infección grave y desenlace fatal ${ }^{23,35,36}$. La positividad de la RPC en sangre puede anteceder a la presencia de síntomas en 8 a 21 días $^{37}$. Un autor reporta que $82 \%$ de los pacientes con RPC positiva en sangre tuvieron una evolución fatal ${ }^{36}$. La técnica de RPC cuantitativa es indicativa de actividad y ha mostrado ser útil en monitorizar la respuesta a la terapia específica $^{38,39}$. Varios reportes han asociado una carga viral alta con sepsis y un curso sistémico fatal; sin embargo, aún faltan estudios para determinar qué valores definen la presencia de enfermedad de mal pronóstico ${ }^{15,37,39}$. Actualmente, en algunos centros se vigila, de rutina, la infección por ADV en pacientes post TPH y se considera el uso de antivirales en los pacientes con evidencias de infección ${ }^{21,36}$. Además de la RPC en sangre, el aislamiento de ADV de múltiples sitios también se considera un fuerte predictor para el desarrollo de infección diseminada ${ }^{6,19}$.

No existen agentes probadamente efectivos para tratar la infección por ADV y no se han realizado estudios prospectivos randomizados para probar la real eficacia de los antivirales. En la literatura científica sólo existen reportes de casos asilados y pequeñas series de casos clínicos. Se han publicado casos anecdóticos de éxito con el uso de ribavirina intravenosa, un análogo de guanosina ${ }^{22,32}$. Pacientes con cistitis hemorrágica y neumonitis han presentado una evolución satisfactoria con su uso $\mathrm{s}^{33,40,41}$. Sin embargo, varios autores han informado que en infecciones sistémicas no se observa una mejoría clínica ni reducción de la carga viral ${ }^{22,42}$. En desarrollo se encuentran estudios del uso de ribavirina en forma profiláctica ${ }^{43}$.

Con cidofovir, un nucleósido monofostato análogo de citosina que inhibe la ADN polimerasa, se han reportado casos exitosos ${ }^{14,33,44-46}$. Su principal limitación es la nefrotoxicidad; debe ser administrado en conjunto con probenecid para disminuir el riesgo de daño renal. En pacientes con TPH e infección diseminada se ha demostrado una mejoría del cuadro clínico y disminución de la mortalidad ${ }^{21,24,33,45,46}$. Se obtiene también supresión viral, aunque algunos autores refieren que sólo se logra con una terapia prolongada ${ }^{14,24}$. Una reciente publicación destaca la importancia de iniciar terapia precozmente, con cargas virales mayores a 100.000 copias/mL, lo que permite adelantarse en promedio 20 días a la aparición de los síntomas ${ }^{24}$. Aún no está claro si todas las infecciones por ADV deben tratarse, pues se han descrito pacientes con recuperación espontánea ${ }^{47}$. Algunos autores refieren que la terapia antiviral siempre debe ser acompañada, en lo posible, de una disminución de la immunosupresión para reducir la mortalidad ${ }^{34}$.

La importancia de la recuperación de la respuesta específica a ADV mediada por LT en la mejoría de estos pacientes ha llevado a desarrollar la immunoterapia "adoptiva", con infusión de LT específicos ${ }^{22,48}$. Se han reportado casos exitosos con tratamiento con linfocitos del donante o de hermano HLA idéntico ${ }^{33,49}$. Esta sería también una opción para aquellos pacientes que no puedan recibir cidofovir y no reconstituyen su sistema inmune ${ }^{24}$. El rol de la inmunoglobulina endovenosa aún no está claro. Algunos autores confían que en el futuro próximo se podría tener éxito con inmunoterapia específica anti- ADV post TPH profiláctica. Como un primer paso en esta línea, estos investigadores generaron linfocitos CD4 citotóxicos ex-vivo infectando células dendríticas inmaduras con $\mathrm{ADV}^{50}$. Se ha planteado además el seguimiento de la respuesta de células T específicas para definir la terapia de la infección por ADV en los pacientes sometidos a $\mathrm{TPH}^{22}$

Debido al creciente grupo de pacientes inmunocomprometidos en la práctica clínica, se debe tener un alto índice de sospecha de infección por ADV en ellos, especialmente en los niños sometidos a TPH. Ante la presencia de síntomas gastrointestinales, fiebre, neumonía, cistitis o hepatitis, se deben tomar muestras para identificar ADV, especialmente si el estudio de 
bacterias, hongos, CMV y VHS es negativo. La muestra con mayor rendimiento diagnóstico en la infección precoz es la deposición. La terapia precoz con antivirales podría evitar cursos fatales, pero se necesitan más estudios para conocer mejor el rol de la terapia antiviral en estas circunstancias clínicas, así como aclarar la utilidad de realizar vigilancia activa de esta infección en niños de alto riesgo y de iniciar terapia previa al desarrollo de síntomas (pre emptive therapy). Casos como éste además, destacan la necesidad de disponer en nuestro país de antivirales para tratar en forma oportuna estas graves infecciones.

Agradecimientos. A Marcela Ferrés y el personal del Laboratorio de Infectología y Virología Molecular, Hospital Clínico Pontificia Universidad Católica de Chile, donde se realizó parte del estudio virológico de este paciente.

\section{Resumen}

Las infecciones graves por adenovirus (ADV) tienen una importancia creciente en pacientes inmunocomprometidos, en especial en niños sometidos a trasplante de precursores hematopoyéticos (TPH). Se reporta un caso de infección por ADV inicialmente gastrointestinal y luego diseminada, de curso fatal, en un niño de 12 años, post TPH. El diagnóstico se confirmó mediante aislamiento viral y detección de genoma viral en tejidos y sangre. Se revisan los principales aspectos de la infección por ADV, su diagnóstico y las posibilidades terapéuticas. Este caso debe alertar a los médicos clínicos para sospechar y estudiar este agente viral en pacientes de alto riesgo y enfatiza la importancia de disponer en Chile de antivirales para su tratamiento.

\section{Referencias}

1.- Chuang Y Y, Chiu C H, Wong K S, Huang J G, Huang Y C, Chang L Y, et al. Severe adenovirus infection in children. J Microbiol Immunol Infect 2003; 36: 37-40.

2.- Rocholl C, Gerber K, Daly J, Pavia A T, Byington $\mathrm{Cl}$. Adenoviral infections in children: the impact of rapid diagnosis. Pediatrics 2004; 113: e51-6.

3.- Larrañaga C, Kajon A, Villagra E, Avendaño L F. Adenovirus surveillance on children hospitalized for acute lower respiratory infections in Chile (1988-1996). J Med Virol 2000; 60: 342-6.

4.- Wasserman R, August C S, Plotkin S A. Viral infections in pediatric bone marrow transplant patients. Pediatr Infect Dis J 1988; 7: 109-15.

5.- Shields A F, Hackman R C, Fife K H, Corey L, Meyers J D. Adenovirus infection in patients undergoing bone marrow transplantation. N Engl J Med 1985; 312: 529-33.

6.- Flomemberg P, Babbitt J, Drobyski W R, Ash R C, Carrigan D R, Sedmak G V, et al. Increasing incidence of adenovirus in bone marrow recipients. J Infect Dis 1994; 169: 775-81.

7.- Blanke C, Clark C, Broun E R, Tricot G, Cunningham I, Cornetta K, et al. Evolving pathogens in allogeneic bone marrow transplantation: increased fatal adenoviral infections. Am J Med 1995; 99: 326-8.

8.- $\mathrm{Xu} \mathrm{W,} \mathrm{McDonough} \mathrm{M,} \mathrm{Erdman} \mathrm{D.} \mathrm{Species-}$ specific identification of human adenoviruses by a multiplex PCR assay. J Clin Microbiol 2000; 38: 4114-20.

9.- Kajon A E, Larrañaga C, Suárez M, Wadell G,
Avendaño L F. Genome type analysis of Chilean adenovirus strains isolated in a children's hospital between 1988 and 1990 . J Med Virol 1994; 42: 16-21.

10.- Palomino M A, Larrañaga C, Avendaño L F. Hospital-acquired adenovirus $7 \mathrm{~h}$ infantile respiratory infection in Chile. Pediatr Infect Dis J 2000; 19: 527-31.

11.- Baldwin A, Kingam H, Darville M, Foot A B, Grier D, Cornish JM, et al. Outcome and clinical course of 100 patients with adenovirus infection following bone marrow transplantation. Bone Marrow Transplant 2000; 26: 1333-8.

12.- La Rosa A M, Champlin R E, Mirza N, Gajewski J, Giralt S, Rolston K V, et al. Adenovirus infections in adult recipients of blood and marrow transplants. Clin Infect Dis 2001; 32: 871-6.

13.- Runde V, Ross S, Trenschel R, Lagemann E, Basu O, Renzing-Köhler K, et al. Adenoviral infection after allogeneic stem cell transplant (SCT): report on 130 patients from a single SCT unit involved in a prospective multicentre surveillance study. Bone Marrow Transplant 2001; 28: 51-7.

14.- Hoffman J A, Shab A J, Ross L A, Kapoor $\mathrm{N}$. Adenoviral infections and a prospective trial of cidofovir in pediatric hematopoietic stem cell transplantation. Biol Blood Marrow Transplant 2001; 7: 388-94.

15.- Walls T, Shankar A G, Shingadia D. Adenovirus: an increasingly important pathogen in pediatric bone marrow transplant patients. Lancet Infect Dis 2003; 3: $79-86$

16.- Zahradnik J M, Spencer M J, Porter D D. Adenovirus infection in the immunocompromised patient. Am J Med
1980; 68: 725-32.

17.- Krilov L R, Rubin L G, Frogel M, Gloster E, Ni K, Kaplan M, et al. Disseminated adenovirus infection with hepatic necrosis in patients with human immunodeficiency virus infection and other immunodeficiency states. Rev Infect Dis 1990; 12: 303-7.

18.- Hierholzer J C. Adenovirus in the immunocompromised host. Clin Microbiol Rev 1992; 5: 262-74.

19.- Michaels M G, Green M, Wald E R, Starzl T E. Adenovirus infection in pediatric liver transplant recipients. J Infect Dis 1992; 165: 170-4.

20.- Leruez-Ville M, Minard V, Lacalille F, Buzyn A, Abachin E, Blanche S, et al. Realtime blood plasma polymerase chain reaction for management of disseminated adenovirus infection. Clin Infect Dis 2004; 38: 45-52.

21.- Kampmann B, Cubitt D, Walls T, Naik P, Depala M, Samarasinghe S, et al. Improved outcome for children with disseminated adenoviral infection following allogeneic stem cell transplantation. Br J Haematol 2005; 130: 595-603.

22.- Feuchtinger $T$, Lang $P$, Handgretinger $R$. Adenovirus infection after allogeneic stem cell transplantation. Leukemia \& Lymphoma 2007; 48: 244-55.

23.- Chakrabarti S, Mautner V, Osman H, Collingham K E, Fegan C D, Klapper P E, et al. Adenovirus infection following allogeneic stem cell transplantation: the incidence and outcome in relation to graft manipulation, immunosupression and immune recovery. Blood 2002; 100: 161927.

24.- Neofytos D, Ojha A, Mookerjee B, 
Wagner J, Filicko J, Ferber A, et al. Treatment of adenovirus disease in stem cell transplant recipients with cidofovir. Biol Blood Marrow Transplant 2007; 13: 74-81.

25.- Ison M. Adenovirus infections in transplant recipients. Clin Infect Dis 2006; 43: 331-39.

26.- Hale G, Heslop H, Krance R, Brenner M, Jayawardene D, Srivastava D. Adenovirus infection after pediatric bone marrow transplantation. Bone Marrow Transplant 1999; 23: 277-82.

27.- Wang $W H$, Wang $H$ L. Fulminant adenovirus hepatitis following bone marrow transplantation. Arch Pathol Lab Med 2003; 127: e246-8.

28.- Carmichael G P, Zahradnik J M, Moyer G H, Porter D D. Adenovirus hepatitis in an immunosuppressed adult patient. Am J Clin Pathol 1979; 71: 352-5.

29.- Kaur B, Gottardo N G. A rare case of adenoviral fulminant hepatic necrosis after chemotherapy. Pediatr Hematol Oncol 2002; 19: 361-71.

30.- Cames B, Rahier J, Burtomboy G, de Ville de Goyet J, Reding R, Lamy M, et al. Acute adenovirus hepatitis in liver transplant recipients. J Pediatr 1992; 120: 33-7.

31.- Horvath J, Palkonyay L, Weber J. Group C adenovirus DNA sequences in human lymphoid cells. J Virol 1986; 59: 189-92.

32.- Heemskerk B, Lankester AC, Van Vreeswijk T, Beersma M, Claas E C, Veltrop-Duits LA, et al. Immune reconstitution and clearance of human adenovirus viremia in pediatric stem-cell recipients. J Infect Dis 2005; 191: 520-30.

33.- Bordigoni P, Carret A S, Venard V, Witz F, Le Faou A. Treatment of adenovirus infections in patients undergoing allogeneic hematopoietic stem cell transplantation. Clin Infect Dis 2001; 32: 1290-7.

34.- Sivaprakasam P, Carr T, Coussons M, Khalid T, Bailey A, Guiver M, et al. Improved outcome from invasive adenovirus infection in pediatric patients after hematopoietic stem cell transplantation using intensive clinical surveillance and early intervention. J Pediatr Hematol Oncol
2007; 29: 81-85.

35.- Echavarria M, Forman M, Van Tol M J, Vossen J M, Charache P, Kroes A C. Prediction of severe disseminated adenovirus infection by serum PCR. Lancet 2001; 358: 384-5.

36.- Lion $\mathrm{T}$, Baumgartinger $\mathrm{R}$, Watzinger $\mathrm{F}$, Matthes-Martin S, Suda M, Preuner S, et al. Molecular monitoring of adenovirus in peripheral blood after allogeneic bone marrow transplantation permits early diagnosis of disseminated disease. Blood 2003; 102; 1114-20.

37.- Seidemann K, Heim A, Pfister E, Köditz H, Beilken A, Sander A, et al. Monitoring of adenovirus infection in pediatrics transplant recipient by quantitative PCR: report of six cases and review of the literature. Am J Transplant 2004; 4: 2102-08.

38.- Teramura T, Naya M, Yoshihara T, Morimoto A, Imashuku S. Quantitative detection of serum adenovirus in a transplant recipient. Lancet 2002; 359: 1945.

39.- Schilham M, Claas E, Zaane W, Van Zaane W, Heemskerk B, Vossen J M, et al. High levels of of adenovirus DNA in serum correlate with fatal outcome of adenovirus infection in children after allogeneic stemcell transplantation. Clin Infect Dis 2002; 35: 526-32.

40.- Shetty A K, Gans H A, So S, Millan M T, Arvin A M, Gutiérrez K M. Intravenous ribavirin therapy for adenovirus pneumonia. Pediatr Pulmonol 2000; 29: 69-73.

41.- Miyamura K, Hamaguchi M, Taji H, Kanie T, Kohno A, Tanimoto M, et al. Successful ribavirin therapy for severe adenovirus hemorrhagic cystitis after allogeneic marrow transplant from close HLA donors rather than distant donors. Bone Marrow Transplant 2000; 25: 545-8.

42.- Gavin P J, Katz B Z. Intravenous ribavirin treatment for severe adenovirus disease in immunocompromised children. Pediatrics 2002; 1100: e9.

43.- Greil J, Bader P, Lang P, Beck R, Feuchtinger T, Niethammer D.
Prophylactic ribavirin treatment prevents adenovirus infection in pediatric stem cell transplant recipients [abstract]. Blood (Suppl) 2003; 102: 449B.

44.- Ribaud P, Scieux C, Freymuth F, Morinet F, Gluckman E. Successful treatment of adenovirus disease with intravenous cidofovir in an unrelated stem-cell transplant recipient. Clin Infect Dis 1999; 28: $690-1$.

45.- Legrand F, Berrebi D, Houhou N, Freymuth F, Faye A, Duval M, et al. Early diagnosis of adenovirus infection and treatment with cidofovir after bone marrow transplantation in children. Bone Marrow Transplant 2001; 27: 621-6.

46.- Yusuf U, Hale G, Carr J, Gu Z, Benaim E, Woodard P, et al. Cidofovir for the treatment of adenoviral infection in pediatric hematopoietic stem cell transplant patients. Transplantation 2006; 81: 1398404.

47.- Walls T, Hawrami K, Ushiro-Lumb I, Shingadia D, Saha V, Shankar A G. Adenovirus infection after pediatric bone marrow transplantation: is treatment always necessary? Clin Infect Dis 2005; 40: 12449.

48.- Feuchtinger T, Matthes-Martin S, Richard C, Lion T, Fuhrer M, Hamprecht K, et al Safe adoptive transfer of virus-specific T-cell immunity for the treatment of systemic adenovirus infection after allogeneic stem cell transplantation $\mathrm{Br} \mathrm{J}$ Haematol 2006; 134: 64-76.

49.- Hromas R, Cornetta K, Srour E, Blanke C, Broun E R. Donor leukocyte infusion as therapy of life-threatening adenoviral infections after T-cell-depleted bone marrow transplantation. Blood 1994; 84 : 1689-90.

50.- Regn S, Raffegerst S, Chen X, Schendel D, Kolb H J, Roskrow M. Ex vivo generation of cytotoxic $\mathrm{T}$ lymphocytes specific for one or two distinct viruses for the prophylaxis of patients receiving an allogeneic bone marrow transplant. Bone Marrow Transplant 2001; 27: 53-64. 